\title{
PLASMONIC NANOPARTICLE-BASED PROTEIN DETECTION BY OPTICAL SHIFT OF A RESONANT MICROCAVITY
}

Miguel A. Santiago-Cordoba ${ }^{1}$, Svetlana V. Boriskina ${ }^{2}$, Frank Vollmer ${ }^{*}, 3,4$, Melik C. Demirel ${ }^{*}, 1,3$

1. Department of Engineering Science, and Materials Research Institute, Pennsylvania State University, University Park, Pennsylvania, 16802, USA.

2. Department of Chemistry and Electrical and Computer Engineering, Boston University, Boston, Massachusetts, 02215, USA

3. Wyss Institute for Bioinspired Engineering, Harvard, Cambridge, Massachusetts 02138, USA.

4. Current address: Max Planck Institute for the Science of Light, Laboratory of Biophotonics \& Biosensing, 91058 Erlangen, Germany.

Keywords: Whispery gallery modes (WGM), label-free biosensing, protein detection, bovine serum albumin (BSA), Au nanoparticle, high Q microcavity, optical resonance

* Correspondence should be addressed to MCD and FV. Email: mdemirel@engr.psu.edu, frank.vollmer@mpl.mpg.de 


\section{ABSTRACT}

We demonstrated a biosensing approach which, for the first time, combines the high-sensitivity of whispering gallery modes (WGM) with a metallic nanoparticle based assay. We provided a computational model based on generalized Mie theory to explain the higher sensitivity of protein detection through Plasmon enhancement. We quantitatively analyzed the binding of a model protein (i.e., BSA) to gold nanoparticles from high-Q WGM resonance frequency shifts, and fit the results to an adsorption isotherm, which agrees with the theoretical predictions of a twocomponent adsorption model. 
A whispering-gallery-mode (WGM) biosensor uses high-Q optical resonances to directly detect the binding of molecules and nanoparticles from a frequency shift signal ${ }^{1-4}$. The WGM biosensing approach is highly sensitive, down to the single virus and nanoparticle level ${ }^{5,} 6$. However, rapid sample analysis (given the small sensing area), varied surface functionalization of monolithic sensor arrays, as well as specific detection against a complex backround are some of the challenges in this field7, 8 . Nanoparticle (NP) biosensing platforms such as those based on gold $(\mathrm{Au}) \mathrm{NPs}$, on the other hand, may overcome some of these challenges by providing a large sensing area, rapid mixing of the analyte, and the possibility for extracting NPs from a complex sample for subsequent optical analysis ${ }^{9-11}$.

In this letter, we combine the advantages of a NP-based assay with the high sensitivity of a WGM biosensor to determine the amount of protein bound to NPs by analyzing frequency/wavelength shifts of hybrid photonic-plasmonic modes. We use $\mathrm{Au}$ NPs to nonspecifically adsorb Bovine Serum Albumin (BSA) protein from a phosphate buffered saline (PBS) solution. After incubation in the BSA solution (SeraCare Life Sciences, Inc), the NPs are filtered, and immobilized on an anodic aluminum oxide (AAO) capture-membrane. Subsequently, the dry NP layer is interrogated by observing high-Q WGM resonance frequency shifts that occur after evanescent coupling of a microsphere cavity to NPs in the layer. By analyzing WGM resonance frequency shifts for NPs exposed to different BSA concentration levels, we find that the adsorption isotherm agrees with a two-component adsorption model. We show picomolar sensitivity levels for this approach which decouples WGM transducer from (NP) recognition element.

The method for preparation of the Au-NP solution with BSA, and capture of the NP layer on the AAO membrane (AAO, Whatman Inc.) by vacuum suction are shown schematically in Figure 1a. The preparation of the Au NPs was done following a modification of the Lee and Meisel's method as described by Kruszewski et al. ${ }^{12}$ Figure $1 \mathrm{~b}$ shows the top surface electron micrograph of bare AAO substrate and the Au NPs captured on the AAO membrane. 
The resonant microsphere cavity structure and its orientation with respect to the Au NPs captured on the AAO membrane are illustrated in Figure 1c. We used a well established WGM setup that monitors WGM resonances of a tapered-fiber-coupled microsphere in real-time ${ }^{13}$. We determined wavelengths shifts associated with coupling of WGM to the NPs by recording and subtracting WGM resonance wavelengths measured for the cavity held in air, and then in contact with the NP layer. Typical WGM spectra before and after coupling to Au NP layer is shown in Figure 1d. We record the shift for two WGMs centered around $\sim 632.014 \mathrm{~nm}$ (Peak 1) and $\sim 632.016 \mathrm{~nm}$ (Peak 2) wavelengths.

The silica microsphere (diameter of $450 \pm 10 \mu \mathrm{m}$ ) probes the NP layer via its evanescent field which extends about $L \sim 50 \mathrm{~nm}$ at $633 \mathrm{~nm}$ wavelength ( $\mathrm{\sim} \sim 80 \mathrm{~nm}$ at $1060 \mathrm{~nm}$ wavelength) outward from the microsphere surface, where $\mathrm{L}=\lambda\left[4 \pi\left(\mathrm{n}_{\text {eff,sphere- }}^{2} \mathrm{n}_{\text {eff,medium }}\right)^{1 / 2}\right]^{-1}, n_{\text {eff,sphere }} \sim 1.45$ and $n_{\text {eff,medium }} \sim 1$ are the effective refractive indices of the glass microsphere and that of the medium surrounding the NPs (air), and $\lambda$ is the nominal wavelength of the laser ${ }^{14}$. The limited extend of $\mathrm{L}$ ensures that we are probing only the first layer of Au-NPs. Typical Q factors before coupling the microsphere to $\mathrm{Au}-\mathrm{NP}$ are $\sim 3 \times 10^{6}$ at $633 \mathrm{~nm}$ and $\sim 5 \times 10^{6}$ at $1060 \mathrm{~nm}$.

Next we compare the Au NP WGM shift at $\sim 633 \mathrm{~nm}$ to that at $\sim 1060 \mathrm{~nm}$ wavelength to test for signal enhancement due to plasmon coupling. For the same Au NP layer the wavelength shift averaged over different probing locations is larger at $\sim 633 \mathrm{~nm}$ wavelength $\left(\Delta \lambda=1.2 \pm 0.1 \times 10^{-4}\right.$ $\mathrm{nm})$ as compared to $\sim 1060 \mathrm{~nm}$ wavelength $\left(\Delta \lambda=0.8 \pm 0.1 \times 10^{-4} \mathrm{~nm}\right)$. Coupling WGM to NP plasmon resonance $(\sim 525 \mathrm{~nm})$ can thus enhance signal to noise ratio (SNR).

We performed exact numerical calculations based on the generalized multi-particle Mie theory to reveal the detailed picture of the WGM interaction with the localized surface plasmon (LSP) resonance of the $\mathrm{Au} \mathrm{NP}^{15}, 16$. Experimentally obtained $\mathrm{Au}$ refractive index values were used in the simulations ${ }^{17}$. We selected a smaller sphere $(5 \mu \mathrm{m})$ for the simulation to limit the computation time. Figure 2a shows the simulated values of WGM shifts, $\Delta \lambda / \lambda$, for a single NP bound at the center of the evanescent tail. The hybrid photonic-plasmonic modes due to the coupling of the narrowband WGM with the plasmon resonance feature significant electric field enhancement on 
the NP surface (Figure 2b) and can be efficiently excited in a wide frequency range covering the longer-wavelength slope of the NP LSP resonance peak ${ }^{15}{ }^{16}$. Indeed, our calculations in Figure $2 \mathrm{~b}$ show up to 3 orders-of-magnitude increase of the electric field intensity on the NP surface. Strong WGM coupling to the NP collective electron oscillations at the frequencies close to the NP dipole LSP resonance increases both radiative and dissipative losses, resulting in the decrease of the Q-factors of hybrid modes in the 520-580 $\mathrm{nm}$ frequency range (Figure 2c). This decrease of the structure's ability to accumulate energy from the external excitation field is reflected in the drop of the field intensity close to the NP LSP resonance observed in Figure 2b.

The spatial distributions of the local field intensity near the surface of the microsphere at the resonance wavelength $\lambda \sim 632 \mathrm{~nm}$ in the presence and absence of the Au NP are shown in Figure $2 \mathrm{~d}$ and $2 \mathrm{e}$ respectively. In the frame of the first-order perturbation approximation ${ }^{5}$, fractional wavelength shift of the mode caused by a small protein molecule with a real excess polarizability $\alpha$ at position $\mathbf{r}_{v}$ is directly proportional to the field intensity value at the molecule position $\left|\mathbf{E}\left(\mathbf{r}_{v}\right)\right|^{2}$ and inversely proportional to the energy density integrated over the whole mode volume.

$$
\left(\frac{\Delta \lambda_{r}}{\lambda_{r}}\right) \cong \frac{\alpha / \varepsilon_{0}\left|\mathbf{E}\left(\mathbf{r}_{v}\right)\right|^{2}}{2 \int_{V} \varepsilon_{r}(\mathbf{r})|\mathbf{E}(\mathbf{r})|^{2} d V}
$$

Significant increase of the field in the area accessible by the protein molecules in the microsphere-NP structure (compare Figure $2 \mathrm{~d}$ and $2 \mathrm{e}$ ) is expected to translate into the larger sensitivity of the hybrid WGM-NP sensor. Indeed, our calculations show that adsorption of a single BSA molecule (modeled as a $3.4 \mathrm{~nm}$-radius nanosphere with $n=1.45$ ) at the center of the evanescent field does not produce a detectable shift of the WGM resonance at $\lambda \sim 580 \mathrm{~nm}$. However, the BSA placed in the hot spot created on the WGM-coupled NP causes $\Delta \lambda=1.06 \cdot 10^{-}$ ${ }^{4} \mathrm{~nm}$ shift of the corresponding hybrid resonance. If the BSA molecule were instead placed in the hot spot of the isolated NP alone, it causes $\Delta \lambda=1.497 \cdot 10^{-2} \mathrm{~nm}$ shift of the dipole LSP resonance, due to the small NP mode volume which decreases the value of the denominator in equation (1). However, orders-of-magnitude larger Q-factors of hybrid photonic-plasmonic modes $\left(\mathrm{Q} \sim 10^{6-7}\right)$ in the WGM-NP structure over that of the single NP plasmon resonance increase the hybrid's 
sensor spectral resolution and greatly improves the BSA detection limit as compared to individual, WGM-based or NP-based sensors.

We tested our method with the detection of BSA protein from solution. We incubated NPs with different concentrations of BSA solutions ranging from $\mathrm{pM}$ to $\mu \mathrm{M}$. The Au NPs adsorb BSA protein and after incubation for about three hours, the Au NP-BSA layer is formed on the AAO membrane for subsequent analysis with WGMs. In Figure 3, the WGM wavelength shifts for the two modes (peak 1 and 2, Figure 1d) averaged for different probing locations are plotted. From each measurement we subtracted the wavelength shift for a NP layer exposed to control solution which contains no BSA protein. For BSA concentration higher than $1 \mu \mathrm{M}$, we observed additional increase of the shift signal possibly due to BSA multilayer formation and solution aggregation (Figure 3 inset). We observed slight variation of the shift signal for peak 1 and peak 2 indicating possible influence of the WGM mode number and the polarization on the shift signal.

The isotherm in Figure 3 can be explained by a two-component adsorption model ${ }^{18}$. For each NP subsystem which has $m$ equivalent and independent sites for adsorption, the partition function pertaining to the single NP is $\xi=(1+q \phi)^{m}$, where $q$ is the NP concentration, $\phi=\mathrm{e}^{\mu / \mathrm{kT}}$, $\mathrm{k}$ is the Boltzmann constant, $\mathrm{T}$ is the Temperature, $\mu$ is the binding energy. The average number $\bar{N}$ of bound molecules in the macroscopic two-component system can be estimated from the ensemble of NP subsystems as,

$$
\bar{N}=M \phi\left(\frac{\partial \ln \xi}{\partial \phi}\right)_{T} \text { or } \bar{s}=\frac{\bar{N}}{M}=\phi\left(\frac{\partial \ln \xi}{\partial \phi}\right)_{T}
$$

where $\mathrm{M}$ is the total number of available sites, $\mathrm{s}$ is the average number of adsorbed molecules per NP subsystem with $0 \leq s \leq m$. Inserting the partition function for the NP subsystem in equation 2 yields,

$$
\bar{s}=\phi\left(\frac{\partial}{\partial \phi}\right)(m \ln (1+q \phi))=m \phi \frac{q}{1+q \phi}=m \frac{q \phi}{1+q \phi}
$$

For a single NP or interface $(\mathrm{m}=1)$, the equation 3 reduces to the classic Langmuir adsorption equation. 
In conclusion, we demonstrated that WGM modes can be used to quantitatively probe the amount of BSA protein adsorbed to plasmonic Au NPs. To the best of our knowledge this is the first time that the high-sensitivity of a WGM biosensor is combined with a NP-based assay for optical detection. Future research will focus on increasing SNR by optimizing WGM and NP plasmon resonance, and studying spectroscopically ${ }^{19-21}$ the binding curve between molecules and their NP-binding sites.

\section{ACKNOWLEDGMENT}

We gratefully acknowledge financial support for this work from the Wyss Institute at Harvard, as well as the Rowland institute at Harvard for the cooperation in the use of their facilities.

\section{AUTHOR CONTRIBUTIONS}

MCD and FV planned and supervised the research. MSC, FV and MCD performed the experiments. SVB performed the numerical simulations. All authors contributed to writing and revising the manuscript, and agreed on its final contents.

\section{REFERENCES}

1. K. De Vos, I. Bartolozzi, E. Schacht, P. Bienstman and R. Baets, Opt. Express 15 (12), 7610-7615 (2007).

2. A. J. Qavi and R. C. Bailey, Angew. Chem.-Int. Edit. 49 (27), 4608-4611 (2010).

3. F. Vollmer and S. Arnold, Nat. Methods 5 (7), 591-596 (2008).

4. I. M. White, J. Gohring and X. D. Fan, Opt. Express 15 (25), 17433-17442 (2007).

5. F. Vollmer, S. Arnold and D. Keng, Proc. Natl. Acad. Sci. U. S. A. 105 (52), 2070120704 (2008).

6. J. G. Zhu, S. K. Ozdemir, Y. F. Xiao, L. Li, L. N. He, D. R. Chen and L. Yang, Nat. Photonics 4 (1), 46-49 (2010).

7. Y. G. Kim, S. Moon, D. R. Kuritzkes and U. Demirci, Biosens. Bioelectron. 25 (1), 253258 (2009).

8. A. J. Qavi, A. L. Washburn, J. Y. Byeon and R. C. Bailey, Anal. Bioanal. Chem. 394 (1), 121-135 (2009).

9. K. Fujiwara, H. Watarai, H. Itoh, E. Nakahama and N. Ogawa, Anal. Bioanal. Chem. 386 (3), 639-644 (2006).

10. W. G. Lee, Y. G. Kim, B. G. Chung, U. Demirci and A. Khademhosseini, Adv. Drug Deliv. Rev. 62 (4-5), 449-457 (2010). 
11. K. A. Willets and R. P. Van Duyne, Annu. Rev. Phys. Chem. 58, 267-297 (2007).

12. M. W. Cryankiewicz, Kruszewski, T., Journal of Physics: Conference Series 79 (012013), 1-6 (2007).

13. F. Vollmer, D. Braun, A. Libchaber, M. Khoshsima, I. Teraoka and S. Arnold, Appl. Phys. Lett. 80 (21), 4057-4059 (2002).

14. M. Noto, F. Vollmer, D. Keng, I. Teraoka and S. Arnold, Opt. Lett. 30 (5), 510-512 (2005).

15. S. V. Boriskina and L. Dal Negro, Opt. Lett. 35 (4), 538-540 (2010).

16. S. V. Boriskina and B. M. Reinhard, Proc. Natl. Acad. Sci. U. S. A. 108 (8), 3147-3151 (2011).

17. P. B. Johnson and R. W. Christy, Phys. Rev. B 6 (12), 4370 (1972).

18. T. L. Hill, Introduction to Statistical Thermodynamics. (Addison-Wesley, Reading, P. A., 1960).

19. M. C. Demirel, P. Kao, N. Malvadkar, H. Wang, X. Gong, M. Poss and D. L. Allara, Biointerphases 4 (2), 35-41 (2009).

20. P. Kao, N. A. Malvadkar, M. Cetinkaya, H. Wang, D. L. Allara and M. C. Demirel, Adv. Mater. 20 (18), 3562-3566 (2008).

21. N. A. Malvadkar, G. Demirel, M. Poss, A. Javed, W. J. Dressick and M. C. Demirel, J. Phys. Chem. C 114 (24), 10730-10738 (2010). 


\section{FIGURE CAPTIONS}

Figure 1. a) Schematic of gold nanoparticle (Au NP) preparation. Au NPs were mixed with BSA and incubated at $4{ }^{\circ} \mathrm{C}$ for $3 \mathrm{~h}$ to form BSA-Au NPs. AAO membranes $(100 \pm 10 \mathrm{~nm}$ hole diameter) were soaked for $5 \mathrm{~h}$ in a $\sim 5 \%$ solution of polyethyleneinimide (PEI) to enhance adhesion of Au NPs to AAO membrane. Subsequently, AAO membranes were washed with pure water, and dried in desiccators under vacuum. b) Electron microscopy images of bare AAO and AAO with Au NPs using FEI Philips XL-20 microscope. c) Schematic diagram of the set-up employed to evanescently couple WGMs to the Au nanoparticle layer. Top: WGM transmission spectrum for the microsphere in air. Bottom: WGM spectrum for microsphere in contact with nanoparticles which induce WGM wavelength shift. The microsphere cavity was fabricated by melting the tip of a single mode optical fiber. d) Example of a WGM spectrum in air (dotted line) and after evanescent coupling to a BSA-nanoparticle layer (continuous line).

Figure 2. (a) Spectral shifts of the first-radial-order WGM of a $5 \mu$ m-diameter $\mathrm{SiO}_{2}$ microsphere in air caused by the attachment of $55 \mathrm{~nm}$-diameter Au NP (shown in the inset). (b) Intensity enhancement on the Au NP surface in the presence (red dots) and absence (blue line) of the sphere, in this case under the illumination by a linearly-polarized plane wave. (c) Q-factors of the resonances corresponding to the WGM in the microsphere and hybrid photonic-plasmonic modes in the coupled sphere-NP structure. Spatial electric field intensity distributions at the wavelengths of (d) a hybrid resonance $(\lambda=630.84 \mathrm{~nm})$ and (e) the corresponding WG-mode resonance $(\lambda=630.819 \mathrm{~nm})$ (sphere surface is shown as a dashed line).

Figure 3. WGM measurements at $\sim 633 \mathrm{~nm}$ probing wavelength. Au NPs where incubated with different concentrations of a BSA solution and then immobilized onto the AAO membrane for WGM shift analysis. The inset shows the BSA adsorption at high solution concentrations compared to dilute concentration in a log-linear graph. 
(a)

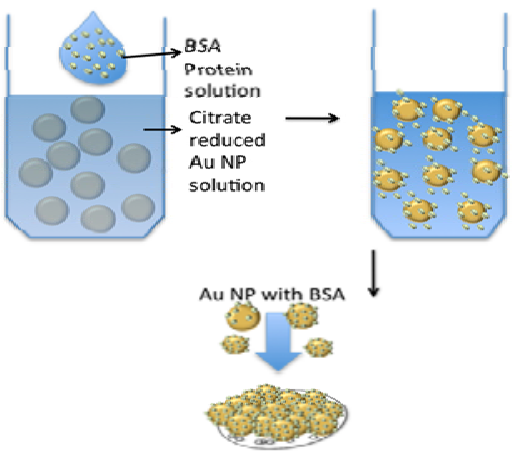

(b)

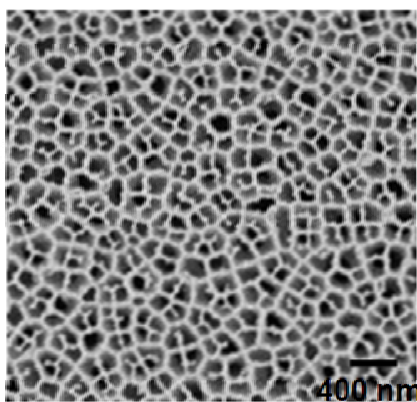

AAO

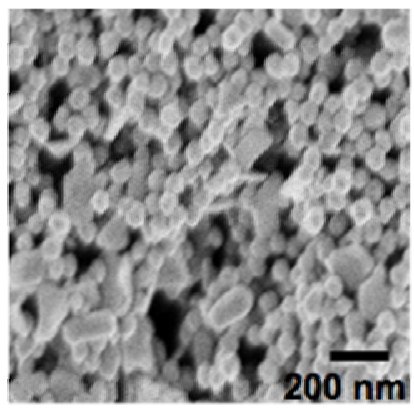

AAO+Au NP (c)

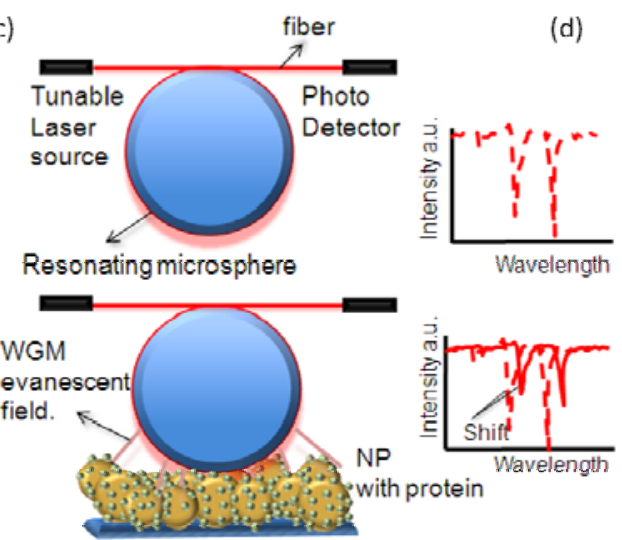

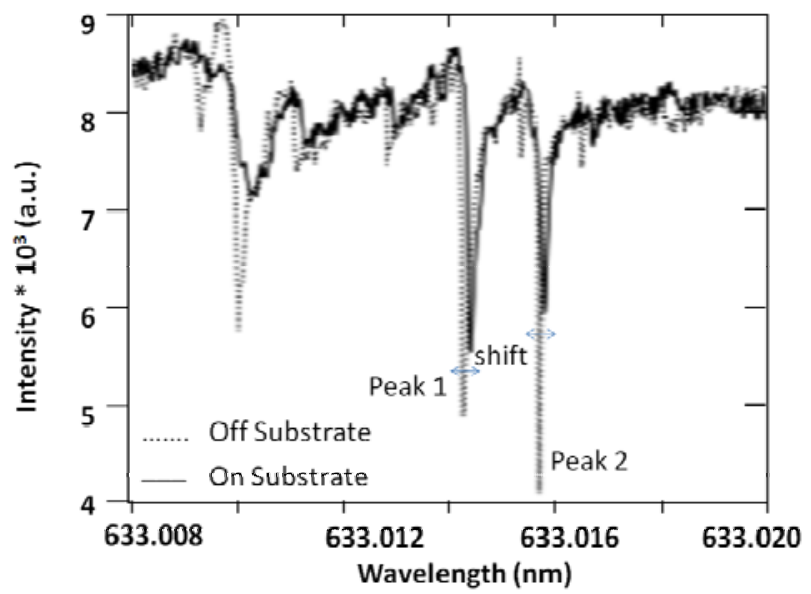



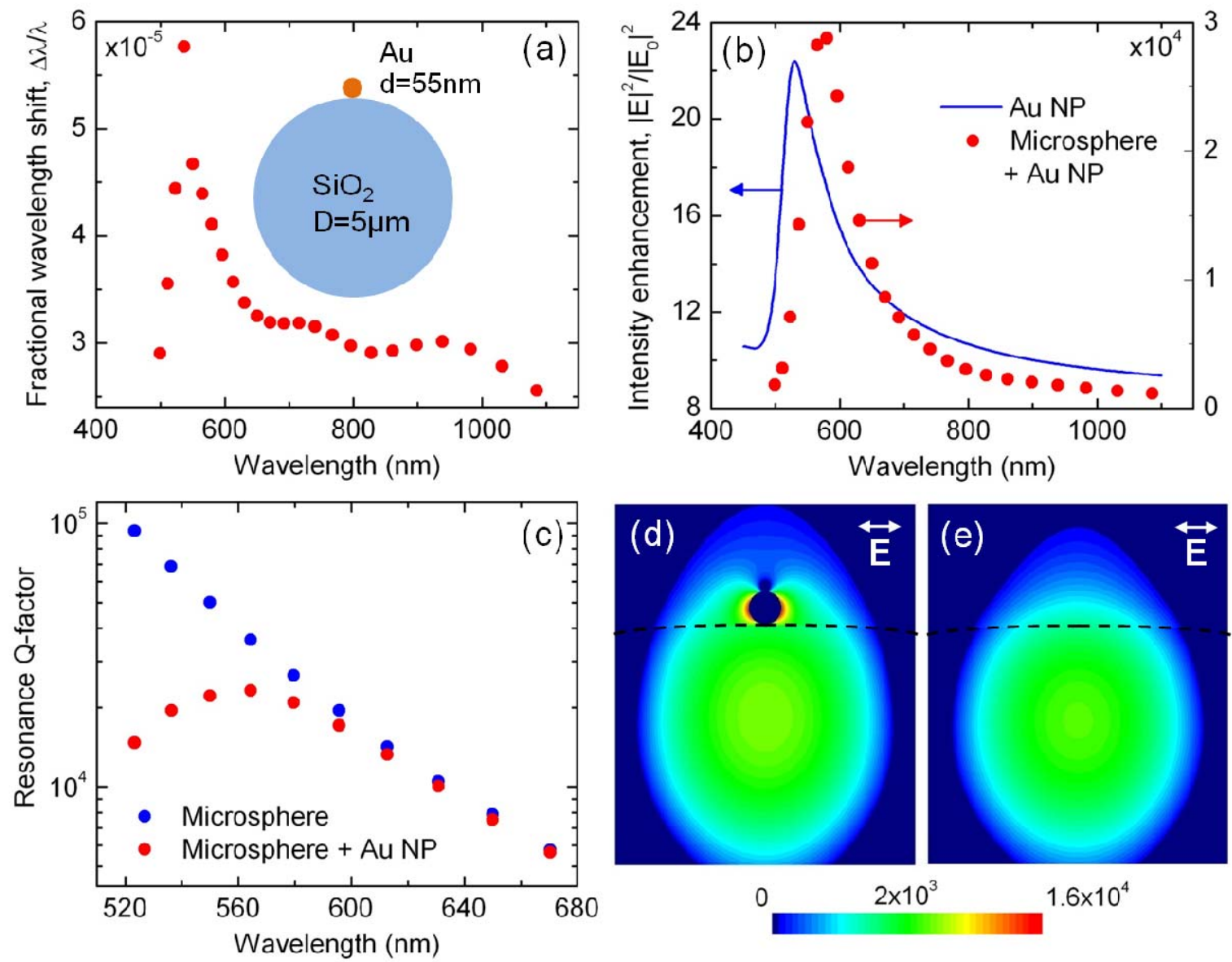


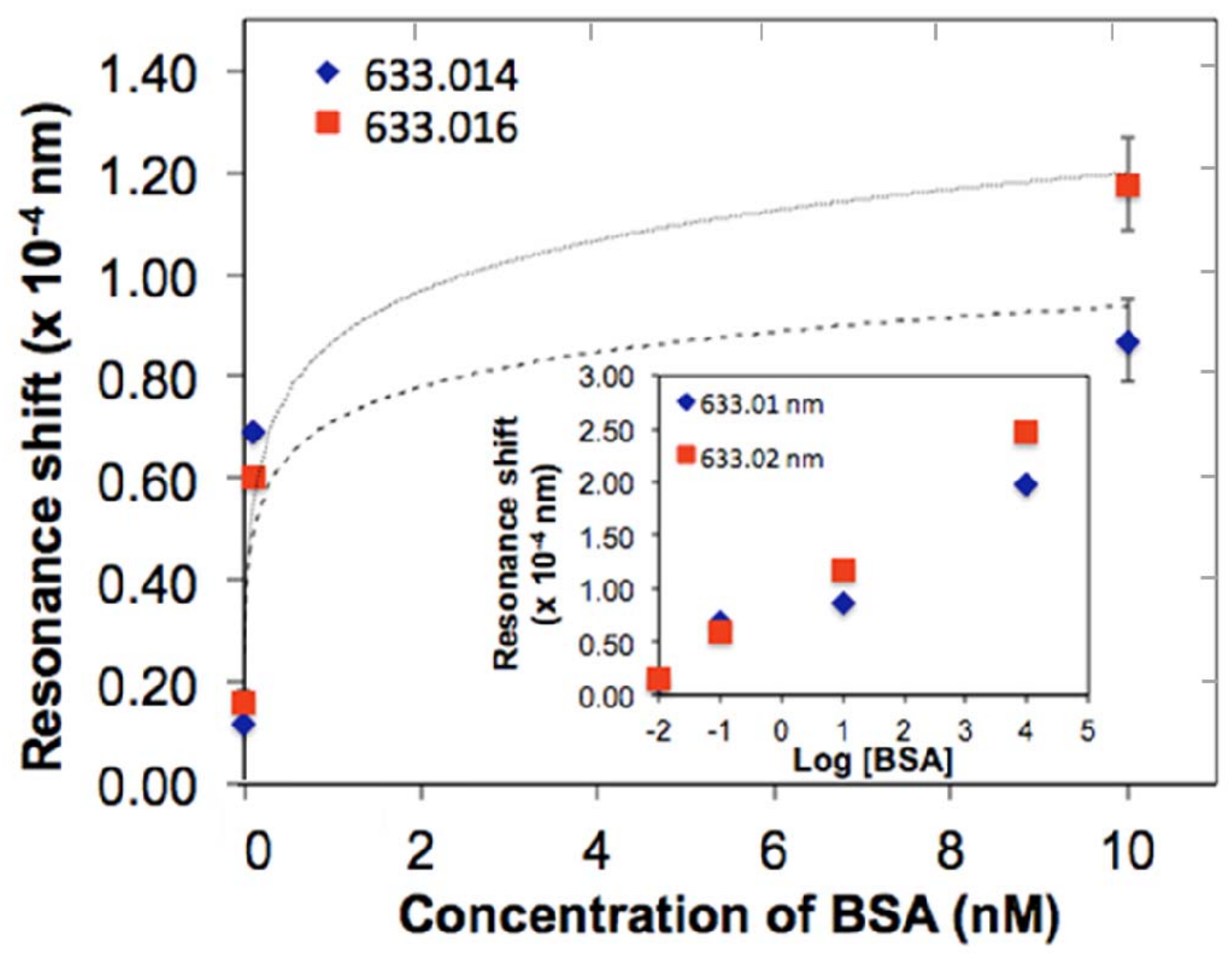

